\title{
DGP-Newsletter Juli 2012
}

\author{
Redaktion: U. Costabel, Essen
}

\section{Entwicklungen in der DGP: Fakten und Zahlen \\ $\nabla$}

Die Entwicklungen in der DGP sind in den letzten Jahren äußerst erfreulich. In diesem Jahr zählt die DGP erstmals über 3000 Mitglieder ( $\bullet$ Abb. 1). Dies bedeutet gegenüber 1990 eine Verdreifachung, alle 10 Jahre sind etwa 1000 Mitglieder hinzugekommen, Ausdruck der zunehmenden Attraktivität der DGP und unseres Fachgebietes.

Der Anteil der Frauen beträgt nur 28,5\%( $\bullet$ Abb.2), 2003 lag er mit 20,6\% aber noch niedriger. Zu erwarten ist hier zukünftig eine weitere Zunahme, da Deutschland inzwischen mehr als $60 \%$ weibliche Medizinstudierende zählt. Hier ist es Aufgabe der Pneumologie, für den weiblichen Nachwuchs vermehrt attraktive Angebote in Klinik und Praxis zu entwickeln, die eine bessere Vereinbarung von Beruf und Familie ermöglichen.

Unter den 3079 Mitgliedern gehören 178 sogenannten assoziierten Berufsgruppen (medizinische Assistenzberufe) an, darunter u.a. PhysiotherapeutInnen und andere Berufsgruppen wie AtmungstherapeutInnen und ArzthelferInnen. In dieser Sektion ist in den nächsten Jahren eine deutliche Steigerung der Mitgliederzahl zu erwarten, da die DGP hier sehr gute Fortbildungsangebote entwickelt hat.
Die weitaus meisten Mitglieder - mit 921 fast ein Drittel - gehören der Sektion „Klinische Pneumologie“ an, gefolgt von den Sektionen „Allergologie und Immunologie“ (525), „Schlafmedizin“ (523), „Intensiv- und Beatmungsmedizin“ (519), „Pneumologische Onkologie“ (488), „Infektiologie und Tuberkulose“ (484) und „Endoskopie“ (451), was die unterschiedlichen klinischen Interessensgebiete repräsentativ widerspiegeln dürfte $(\circlearrowleft$ Abb.3). Zahlenmäßig folgen die Sektionen „Kardiorespiratorische Interaktionen“ (328), „Arbeitsmedizin, Epidemiologie, Umwelt- und Sozialmedizin“ (241), „Prävention und Rehabilitation“ (202), „Pathophysiologie und Aerosolmedizin“ (193), „Zellbiologie“ (135), „Pädiatrische Pneumologie“ (131) und „Thoraxchirurgie“ (95). Diese etwas kleineren Sektionen haben aber eine erhebliche Bedeutung für die Pneumologie, z.T. sind sie auch in eigenen Gesellschaften organisiert wie Arbeitsmedizin, Pädiatrische Pneumologie und Thoraxchirurgie.

Diese Verteilung nach wissenschaftlichen Sektionen zeigt sich auch in etwa in der Anzahl der angenommenen Abstracts auf den Kongressen der beiden letzten Jahre in Dresden und Nürnberg (৫ Abb.4).

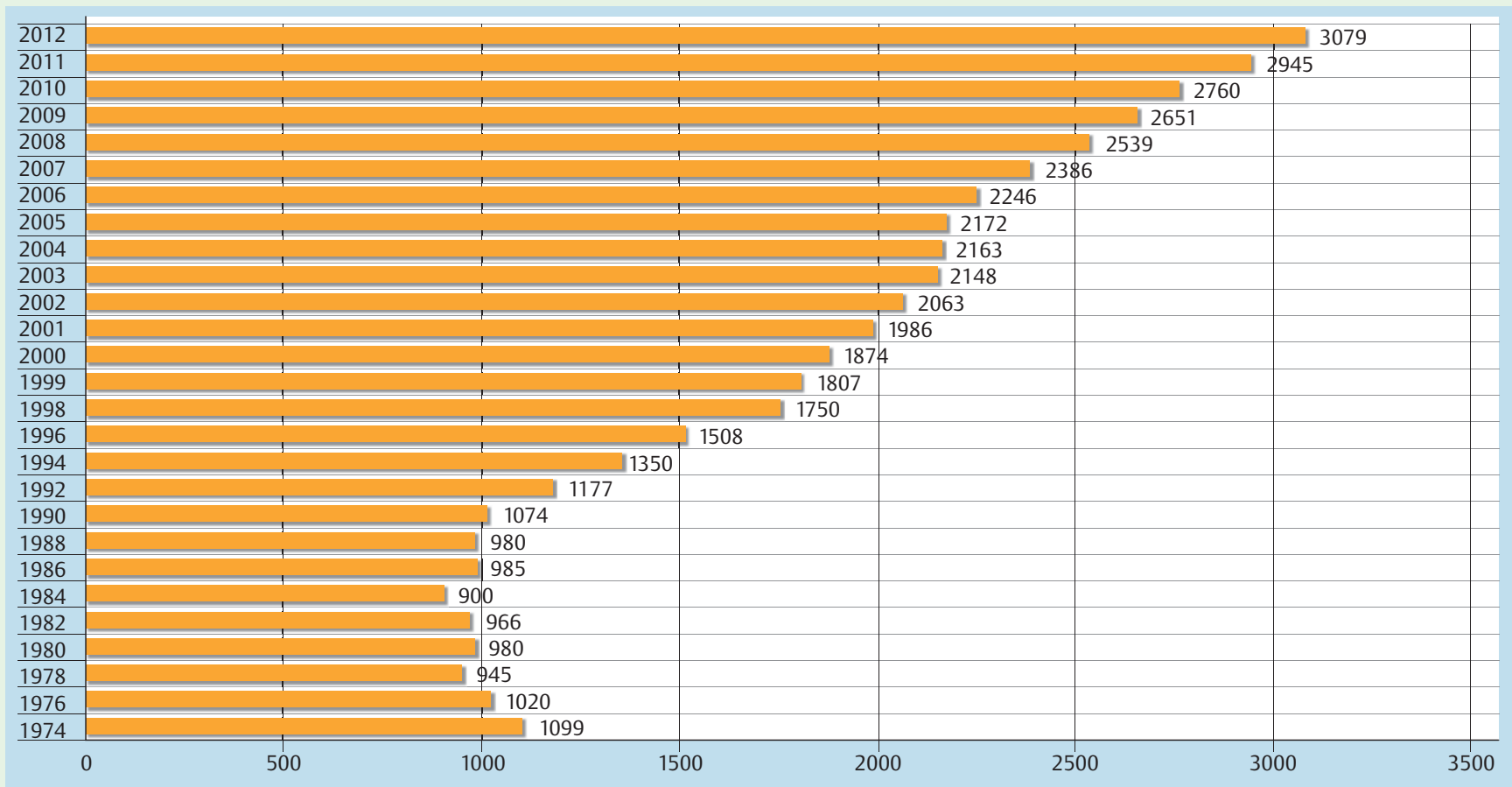

Abb.1 Entwicklung der DGP-Mitgliederzahl seit 1974. 


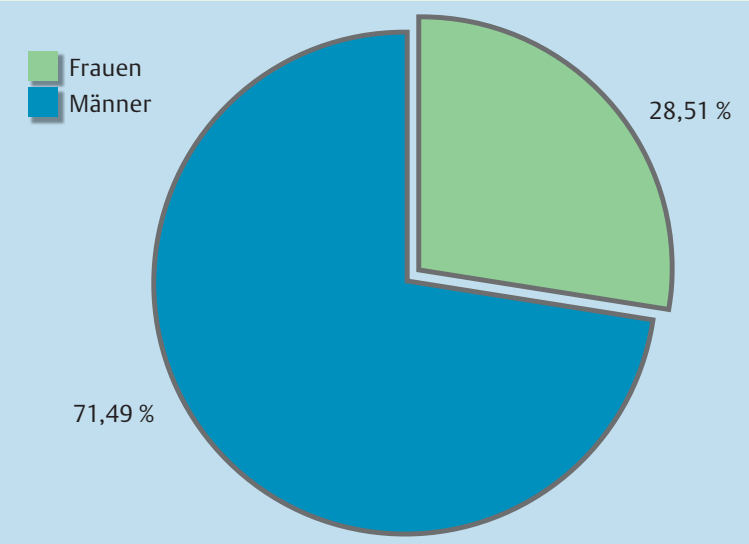

Die Anzahl der Teilnehmer an den Jahreskongressen hat sich in den letzten 10 Jahren mehr als verdoppelt, wobei Dresden 2011 die bisherige Rekordzahl von 3500 Teilnehmern aufweist (๑ Abb.5).

Wie viele Mitglieder wird die DGP in 10 Jahren zählen? 4000 Mitglieder, sollte der Trend anhalten? Wenn es dazu kommen soll, erfordert dies die Fortsetzung der bisherigen Anstrengungen, die Pneumologie in Deutschland auch auf einem international vergleichbar hohen Niveau zu halten und den DGP-Mitgliedern Vorteile zu bieten. Dazu beitragen dürfte die kürzlich erfolgte Gründung von fünf Lungenforschungszentren, aber auch die weitere Professionalisierung der Gesellschaft mit Einstellung einer hauptamtlichen Geschäftsführerin ab August 2012.

Robert Loddenkemper, Berlin

Abb. 2 Prozentualer Anteil der männlichen und weiblichen Mitglieder 2012.

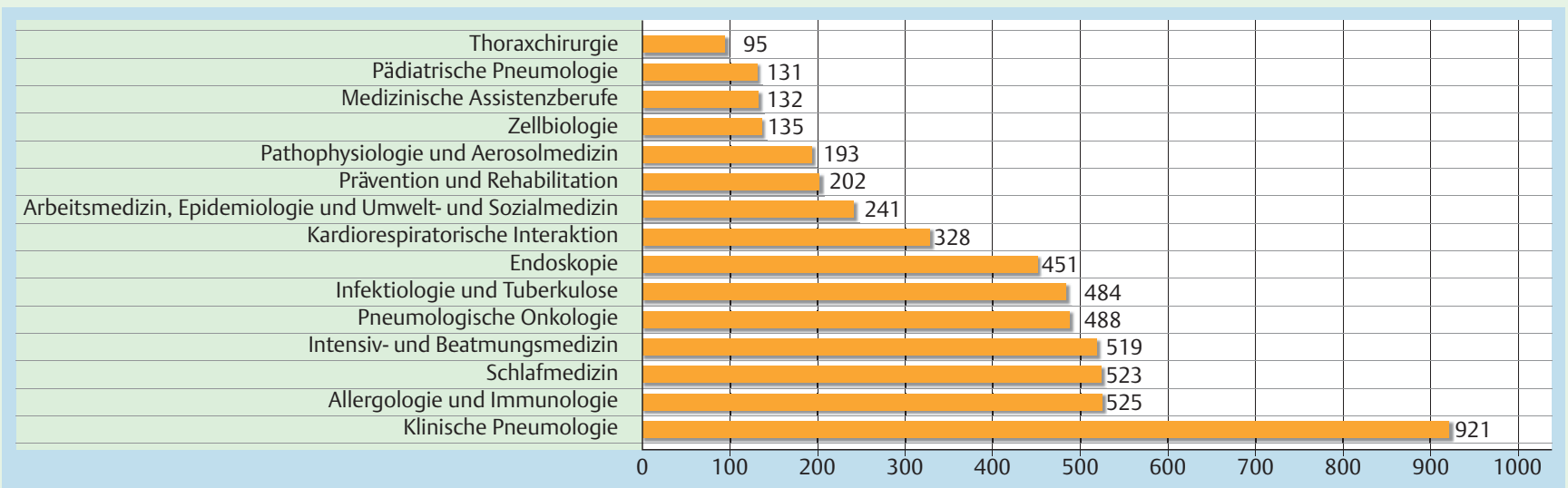

Abb.3 Anzahl der Mitglieder in den wissenschaftlichen Sektionen 2012.

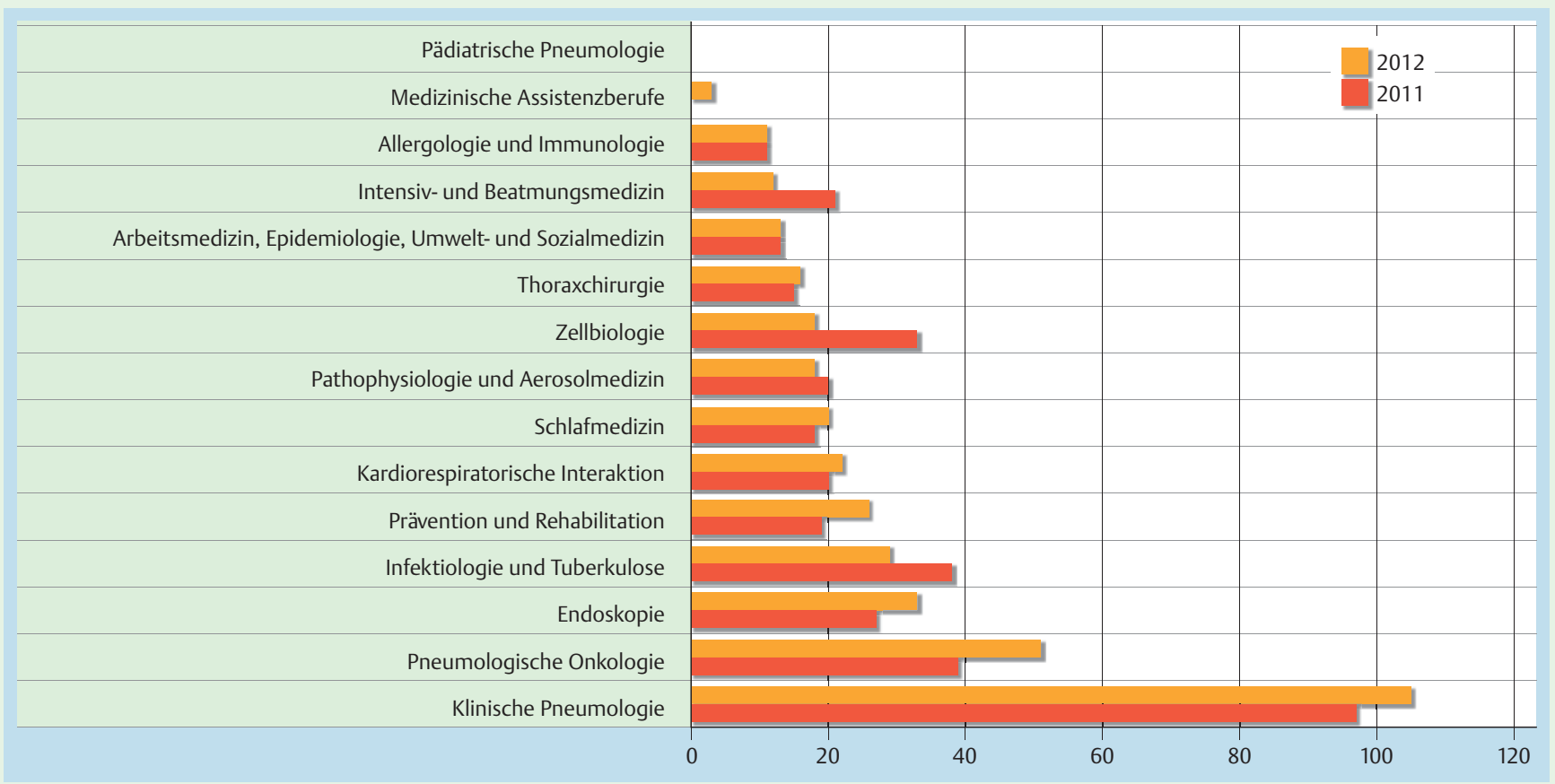

Abb.4 Zahl der angenommenen Abstracts nach wissenschaftlichen Sektionen auf den Kongressen Dresden 2011 und Nürnberg 2012. 


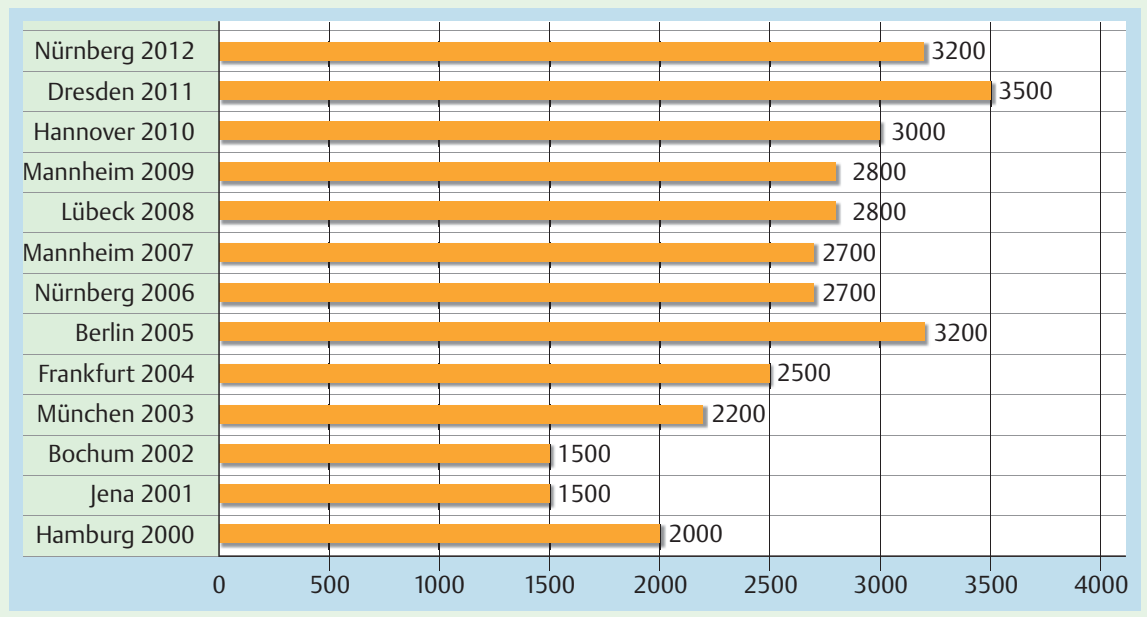

Abb.5 Anzahl der Teilnehmer an den DGP-Jahreskongressen seit 2000.

\section{Arbeitstreffen mit dem Deutschen Netz Rauchfreier Krankenhäuser und Gesundheitseinrichtungen e.v. (DNRfK) \\ $\nabla$}

Pneumologische Kliniken schließen sich zusammen und stärken als Vorreiter den politischen Druck zur Rauchfreipolitik in Deutschland

„Wir haben einen inhaltlichen Auftrag zur Tabakentwöhnung in der Facharztweiterbildung und als Lungenkliniken einen politischen Auftrag, die Tabakentwöhnung in Deutschland umzusetzen." Dies waren Statements auf der Arbeitstagung, zu der am 1. Juni 2012 nach Berlin an die pneumologische Abteilung im Gemeinschaftskrankenhaus Havelhöhe eingeladen worden war. Dass Deutschland in Europa eher auf den hinteren Plätzen im Engagement gegen den inhalativen Tabakkonsum liegt, ist schlimm. „Als pneumologische Kliniken können wir uns im Netz Rauchfreier Krankenhäuser zusammenschließen und so ein eindeutiges Signal an die Öffentlichkeit geben, welche Weichenstellung in der Gesundheitspolitik aus Sicht der Pneumologen gefordert ist."

Prof. R. Loddenkemper stellte den Stand im Antragsverfahren der DGP für eine OPS Ziffer zur Tabakentwöhnung dar, Prof. F. Herth berichtete eindrucksvoll wie sich pneumologische Kliniken für Primärprävention durch regelmäßige Schülerveranstaltungen in der Klinik einbringen können. Dipl. Psych. Peter Lindinger erläuterte praxisnah Prinzipien und das ABC der Raucherberatung mit anschließender telefonischer Nachsorge. Zwei Klinken, das Fachkrankenhaus Coswig (CA Prof. A. Rolle) und das Gemeinschafts- krankenhaus Havelhöhe (Dr. C. Grah), stellten ihre Klinikstrukturen vor, in denen sie in 10 Kategorien eine beratungs- und entwöhnungskompetente Klinik im Silberlevel des Netzwerkes für Rauchfreie Krankenhäuser geschaffen haben. Sie bekamen ebenso wie das HELIOS-Klinikum Schwerin anlässlich der Tagung ihre Urkunde zur Re-Zertifizierung überreicht. Die Koordinatorin des Netzwerkes Frau C. Rustler stellte mit dem Konzept „rauchfrei PLUS“ die Arbeitsweise des Netzwerkes vor. Es wurde hervorgehoben, wie schon allein durch die Mitgliedschaft, die Orientierung an den Standards und der Austausch im Netzwerk - oder auch in den drei Zertifizierungslevels: Bronze, Silber, Gold eine Unterstützung in der internen Klinikkompetenz aufgebaut wird. „Ohne die Zertifizierung hätten wir es in unserem Haus wohl nicht erreicht uns als Rauchfreie Klinik so kompetent gegen dieses große Gesundheitsrisiko zu positionieren.“

In den Grußworten von Univ. Doz. Dr. P. Bölcskei, dem Vorreiter der Tabakentwöhnung in der deutschen Pneumologie, und der Drogenbeauftragten der Bundesregierung Frau M. Dyckmans (MdB), sowie auch der Diskussion wurde deutlich, wie wichtig es ist, dass pneumologische Kliniken politisch und fachlich aktiv das Gesundheitssystem gestalten. Das Netzwerk Rauchfreier Krankenhäuser sollte in Zukunft immer mehr pneumologische Kliniken zu ihren führenden Mitgliedern zählen können! Die Vorträge finden sich unter www.rauchfrei-plus.de zum Downloaden.

Christian Grah, Berlin 